\title{
Inteligencia al servicio de la Ciencia Forense
}

\author{
Intelligence at Service of the Forensic Science
}

\author{
Jesús Fernández Sánchez \\ Analista en investigación criminal
}

\begin{abstract}
Resumen
¿Podemos predecir un perfil de riesgo en la ciencia forense? ¿Podemos estimar sus factores problemáticos? ¿Podemos evitar consecuencias no deseadas? ¿Podemos identificar sus actores implicados? ¿Podemos exponer una idea de trabajo para la evaluación de riesgos en la ciencia forense? ¿Es posible extrapolar metodología de inteligencia para su uso en la ciencia forense? ¿Y la ciencia forense, puede intervenir y coadyuvar a su vez con la inteligencia?

El presente trabajo pone de manifiesto que la inteligencia constituye una vía más en el estudio de la ciencia forense, el conocimiento combinado entre dos disciplinas científicas, su reciprocidad metodológica, su «principio de intercambio doctrinal», puede aportar proyección preventiva respecto de una amenaza, un riesgo, cualquier tipo de incertidumbre o simplemente, proponer acciones encaminadas a evitar la devaluación de un sistema en sus múltiples variantes. Nada ni nadie está exento de un factor problemático, de una situación problemática o de un actor o actores ligados a dicho factor o situación incierta.

Resumiendo, se trata de demostrar que es posible extrapolar el enfoque de la inteligencia desde un punto de vista cualitativo (valor) y exponer la influencia que puede tener en la ciencia forense, no ya desde el aspecto puramente clínico-forense, y sí en aquel aspecto más estratégico, marcado por situaciones de incertidumbre que pueden producirse o provocarse internamente o externamente. Para ello, se antoja necesario conocer el método de trabajo (ciclo de inteligencia) que se sigue para intentar plasmar y evitar un riesgo, el método por sí solo, potenciará cualquier decisión al respecto, sea para formular propuestas de acción de minimización o erradicación de riesgos o para potenciar el rigor y la inexistencia problemática a corto o medio plazo, es decir, ejercerá de filtro ante un situación estimada u objeto problema. También la ciencia forense puede reconvertirse aportando su capacidad a la inteligencia.
\end{abstract}


En suma, la inteligencia es obtenida de la información y sirve a corto plazo para la mejora operativa funcional o de la actividad de la propia ciencia forense. A largo plazo, sirve para la planificación estratégica que se basa en los conceptos más conocidos: debilidades, amenazas, fortalezas y oportunidades (DAFO). En esta planificación, un factor importante a tener en cuenta son las alianzas estratégicas con otras instituciones. En la ciencia forense es obligado citar el convenio de colaboración establecido mediante el Real Decreto 32/2009, de 16 de enero, por el que se aprueba el protocolo de actuación médico forense y de policía científica en sucesos con víctimas múltiples, qué marca un hito en el trabajo en equipo multidisciplinar con un objetivo claro de mejora de los procedimientos y que debería seguir progresando en más aspectos y tipologías delictivas (terrorismo, crimen organizado, delitos violentos etcétera). Este trabajo constituye una aportación más en ese camino iniciado.

\section{Palabras claves}

Ciencias forenses, Arqueología forense, enterramiento clandestino, búsqueda, excavación.

\section{Summary}

Can we predict a profile of risk in the forensic science? Can we estimate his problematic factors? Can we avoid not wished consequences? Can we identify his implied actors? Can we expose an idea of work for the risk evaluation in the forensic science? Is it possible to extrapolate methodology of intelligence for his use in the forensic science? And the forensic science, can it intervene and contribute in turn with the intelligence? Of what way can it infer the forensic science and his disciplines associated in the intelligence?

The present work reveals that the intelligence constitutes one more route in the study of the forensic science, the knowledge combined between two scientific disciplines, his methodological reciprocity, his «beginning of doctrinal exchange», preventive projection can contribute respect of a threat, a risk, any type of uncertainty or simply, propose actions directed to avoiding the devaluation of a system in his multiple variants. Neither nothing nor nobody is exempt from a problematic factor, from a problematic situation or from an actor or actors tied to the above mentioned factor or uncertain situation.

In sum, the intelligence is obtained of the information and serves in the short term for the operative functional improvement or of the activity of the own forensic science. In the long term, it serves for the strategic planning that is based on the concepts more acquaintances: weaknesses, threats, strengths and opportunities (DAFO).

In this planning, an important factor to bearing in mind are the strategic alliances with other institutions. In the forensic science there is forced mentioned the agreement of collaboration established by means of the Royal decree 32/2009, of January 16 , by which there is approved the medical forensic protocol of action and of scientific police in events by multiple victims, 
what marks a milestone in the multidisciplinary teamwork with a clear aim of improvement of the procedures and that should continue progressing in more aspects and tipologías criminal (terrorism, organized crime, violent crimes etc.). This work constitutes one more contribution in this initiated way.

\section{Key words}

Intelligence, forensic science, threat, factor of risk, strategy, futurology, cycle of intelligence, need of intelligence.

\section{Antecedentes previos}

«La solución de un problema requiere previamente ser consciente de él, la percepción del mismo permite la adopción de medidas minimizadoras, paliativas, reactivas y por supuesto, antes que todas ellas, preventivas». (1)

Desde el comienzo del presente trabajo deseo marcar fielmente la importancia que tienen la percepción y la conciencia en toda situación problemática; precisar su entidad, intentar hallar sus causas y analizar sus posibles consecuencias derivadas es fundamental a la hora de abordar la «planificación de una estrategia». Vamos a cuestionar la influencia bipolarmente: como puede intervenir la ciencia forense en el análisis de inteligencia y viceversa, es decir, como puede llegar a interactuar la inteligencia en la ciencia forense, interrelacionando hacia uno u otro lado la capacidad de una y otra al servicio del conjunto.

El trabajo en sí mismo constituye, pues, una innovación en el estudio del delito desde un punto de vista diferente. Me atrevo a pensar que este estudio introductivo de la inteligencia en la ciencia forense y como ésta última se vuelve cíclica para aportar su visión y conocimiento a la primera, conforma un procedimiento multidisciplinar útil y enriquecedor desde la multilateralidad científica que aporta. Llegados aquí surge el primer interrogante: ¿la ciencia forense se encuentra en una situación problemática? Este trabajo no trata de identificar los factores de riesgo que pueden debilitar un «sistema», o que la ciencia forense se encuentre en riesgo, pero no es menos cierto, que toda ciencia, disciplina, método o trabajo no está exento de incidencias problemáticas que pueden llegar a lastrar el raciocinio de la hipótesis o la conclusión, muy ligado al rigor y la credibilidad de acciones, quizás no se percibe o no se tiene conciencia de que el riesgo es presente o pueda ser futurible. La inteligencia puede evaluar el riesgo y erradicar sus consecuencias.

¿Es viable la extrapolación de la inteligencia como método de trabajo en la ciencia forense?

Evidentemente este trabajo va a demostrar que sí lo es, que la ciencia forense puede ir más allá en su estudio empírico, al igual que la inteligencia ha virado también desde sus inicios (amenaza y riesgo de los intereses de la nación, tanto exterior como interior) a la actualidad en la que nos encontramos, con incursión en la obtención de información sobre formas graves de delincuencia, (crimen organizado). Podemos considerar sin lugar a equivocarnos que se ha introducido en el estudio de la delincuencia (análisis criminal). Se deriva una conclusión expresa: la inteligencia aborda el estudio del delito más complejo, aquel que se torna más grave, pero su capacidad, su metodología y su procedimiento no está exento del análisis individualizado de hechos concretos respecto de delitos concretos. Un homicidio o un asesinato puede evaluarse en atención al caso, su investigación, su judicialización y su resolución procesal, pero el conjunto de varios homicidios aún no encontrándose relacionados entre sí, pueden marcar patrones de riesgo a valorar, 
analizar, integrar e interpretar para intentar conocer y prevenir, definiendo medidas disuasorias o soluciones específicas o globales.

Y no sólo eso, puede generar como órgano consultor una visión que advierta que una causa-efecto o que un hecho - consecuencia puede debilitar un sistema en sus diversos y variados aspectos: organizacional, funcional, técnico, perdida de rigor, perdida de credibilidad, etcétera. O de igual modo, evidenciar que ciertos mínimos, lejos de objetivar un método o valorar una ciencia, quizás la agravan por su naturaleza.

La inteligencia al servicio de la ciencia forense, el conocimiento al servicio de la necesidad de conocer (dirección), implica medir lo que se gestiona y la gestión del conocimiento, tanto para saber la dirección en que ir como la difusión del conocimiento (conocimiento y formación) adecuado para cada uno de los actores, la Inteligencia como producto final de la información obtenida (obtención), el análisis al servicio de la estrategia (elaboración) y la estrategia al servicio de la decisión (difusión).

\section{Introducción}

Inteligencia e investigación forense de forma autónoma parecen chocar abiertamente. La visión general y el conocimiento que aporta cada disciplina parece diluirse si tratamos de unirlas, pero no es menos cierto también, que constituye a priori un cóctel cuanto menos interesante.

Es un hecho axiomático que actualmente estamos inmersos en una sociedad globalizada, cambiante y crítica, que busca y anhela un sistema más cualificado, un sistema que ha de relanzarse y sostenerse bajo estrategias competitivas basadas en una estructura global e integral, presidida por criterios tecnológicos garantistas bajo el auspicio I+D, no ya en sentido estricto (certificado) y sí con la calidad que ha de caracterizar un trabajo docente, profesional y pericial que apoye, refuerce y catalogue desde el inicio la toma de decisiones, del tipo académico, profesional o judicial. Es aquí donde la inteligencia fundamenta parte de su razón de ser, anticipando la prevención por encima de la reacción, anticipando la solución por encima del problema futuro; esa es la mayor carta de presentación: «un proyecto calificado en el momento presente capaz de conocer y determinar los riesgos previsibles para proponer planes globales o específicos», cuantificación + cualificación o simplemente, cualificación por encima de cuantificación. Es decir, afianzar la capacidad de esa cualidad desde un planteamiento sencillo, elaborar la idea inicial apostando por invertir la lógica interrogante - respuesta, exponiendo su fortalecimiento precisamente desde una respuesta positiva (percibimos y somos conscientes del problema X, construyamos alrededor de él un «planteamiento evaluador»), evidentemente se trata de dar un plus a la propia ciencia que ejercerá el análisis correspondiente y en contraposición, anticipar una fuga problemática, hallar la debilidad del sistema y por supuesto, anestesiarlo mediante el conocimiento de nuevos métodos aplicados a la identificación problemática y a su solución (acción - reacción).

Un laboratorio puede poseer una certificación oficial (normas ISO, TÜV, FQM, etcétera.) que avale unos resultados periciales y en cambio puede adolecer de capacidades de minimización de riesgos en otros aspectos organizacionales. Es decir, podemos contar con los mejores medios a la hora de abordar un trabajo clínico forense y a su vez, podemos contar con otros intereses o elementos subjetivos que limitan la credibilidad otorgada por los propios estándares de calidad. Un resultado analítico, científico o técnico debe tener la capacidad, objetividad, rigor y dependencia de quien testimonia su labor pericial. Una disminución de actitudes unipersonal (falta formación adecuada, limitada experiencia, intereses privados, etcétera) puede redundar en 
el conjunto de la organización lesionando o debilitando la estrategia adquirida, por lo qué un resultado es más o menos influyente dependiendo de quién lo haga y al servicio de quien lo hace; es decir, minimizar un riesgo no implica exclusivamente ejercer la acción sobre el actor principal, debemos considerar los múltiples actores que intervienen, aunque éstos últimos sean indirectos, pero con capacidad para incidir en el actor directo y principal. Por ello la medición de la capacidad de minimización debe abarcar todo el ámbito competencial de la organización.

La inteligencia aplicada a la ciencia forense, o lo que es lo mismo, la ciencia aplicada a otra ciencia, el conocimiento añadido, no duplicado, el conocimiento secreto o aquel otro no clasificado (abierto) que es capaz de identificar, descifrar, desenmascarar y exponer el objeto problemático: el riesgo. Evidentemente, el método proyecta universalización por cuanto una ciencia, sumada a otra, potenciará necesariamente cualquier decisión, el conocimiento doblemente acreditado, la inteligencia redirigida a la empresa, a la universidad, a la ciencia forense, asumiendo un enfoque dinámico e innovador de una ciencia de carácter exclusivo diseñada en sus orígenes para salvaguardar la seguridad y la defensa nacional. La sinergia de ambas al servicio de la prevención estratégica en cualquier campo de estudio, su extrapolación al interés académico primero y encauzamiento hacia el órgano al que finalmente irá dirigido. Es evidente que el método en sí mismo es potenciador desde un doble posicionamiento: potencia (amplía y/o modifica) decisiones ya tomadas y por otro lado, potencia decisiones embrionarias que hayan de tomarse.

\section{Marco de situación}

Primeramente, antes de avanzar en la exposición, debemos de partir de una premisa básica, o más bien dar respuesta a un interrogante necesario qué disipe dudas desde su inicio, esto es ¿podemos acreditar que todo análisis de inteligencia posee categoría científica? Lógicamente de esta cuestión surgen otras conexionadas:

¿Es por lo tanto una ciencia más?

¿Es referente absoluta y exclusiva de la seguridad y la defensa nacional? ¿Es multidisciplinar y por supuesto cabe apoyar a otras ciencias?.

Sin lugar a dudas, sí es una ciencia más, sin lugar a dudas no es exclusiva de la seguridad y la defensa y sin lugar a dudas puede apoyar a otras ciencias.

Por encima de ello, es incuestionable que el método de trabajo otorga por sí solo garantía y rigor, no en vano, se halla dirigido a reducir la incertidumbre y proponer «soluciones decisorias» en intereses tan vitales como la seguridad y la defensa interior y exterior; por lo tanto, cualquier metodología exclusiva a garantizar dichos intereses, puede ser inclusiva en otras materias objeto de estudio y dar beneficio a otras organizaciones públicas o privadas como apunta en este sentido Gustavo Díaz Matey (2008) (2):

«...la inteligencia debe ser información relevante de algún modo para los que tienen que tomar decisiones, ya sean estatales o pertenecientes a algún tipo de organización no estatal».

"... a pesar de que las definiciones tradicionales de inteligencia catalogan el término únicamente como actividad estatal, hoy en día la mayoría de los expertos consultados son partidarios de una definición más amplia de inteligencia, donde los actores no estatales de diversa índole tengan cabida». 
La ciencia forense ha de tomarse también como información relevante en el ámbito académico, pues el conocimiento aprendido dirimirá futuras decisiones en ese otro ámbito no menos relevante: el auxilio a la justicia.

Todo proyecto de trabajo a priori, ha de marcar unas pautas irrenunciables; en este, creo necesario y conveniente posicionar la inteligencia al mismo nivel que la ciencia forense, categorizar como disciplina científica a una materia que parece escapar a dicha cualidad, es dotarla por sí sola de suficiente peso y credibilidad, por ello, antes de adentrarse en su proceso metodológico, debemos caracterizar ese posicionamiento con las distintas aportaciones que se citan a continuación:

"Todo el proceso de generación de inteligencia es de naturaleza científica, por cuanto obliga al intelecto humano a establecer relaciones, elaborar inferencias, crear escenarios probables, desechar hipótesis imposibles, integrar informaciones, interrogar, validar y explotar satisfactoriamente el contenido de las fuentes, determinar su validez en relación con otras muchas aparentemente inconexas, etc.» (3)

«El carácter multidisciplinar de la inteligencia, que engloba el estudio sistematizado de todas las aportaciones científicas que desde la cátedra, la academia, la milicia y la cultura enriquecen el proceso estatal de adopción de decisiones». (4)

"...la clásica necesidad que motiva el uso de la inteligencia y que se vuelve imperiosa en un mundo abrumado de complejidades e incertidumbres: saber para prever y prever para prevenir» (5)

"Los expertos en inteligencia realizan su misión siguiendo un proceso consolidado de naturaleza científica que transforma datos obtenidos de múltiples fuentes de información (humanas, tecnológicas, abiertas, etc.) en conocimiento especializado que sirve para responder de modo efectivo a las demandas sobre seguridad y sus amenazas por parte de los órganos de decisión política. Al resultado de este proceso se denomina inteligencia». (6)

Expuesta su relación bajo el prisma científico, importante a la hora de calibrar el rigor de toda disciplina, debemos preguntarnos inicialmente si es posible la interrelación de ambas capacidades, inteligencia y ciencia forense:

¿Qué ocurre si mezclamos dos campos de trabajo para aprovechar las virtudes de uno sobre el otro?

¿Pueden llegar a ser compatibles?

¿Es la ciencia forense una disciplina exenta de riesgos?

¿Podemos mejorar sus capacidades futuras?

¿Podemos erradicar sus posibles lagunas?

¿Podemos dotarla de una mayor carga objetiva manteniendo los resultados científicos que ya la caracterizan?

O por el contrario,

¿Los riesgos son inexistentes?

¿Sus capacidades se encuentran sobradamente encasilladas?

¿Carece de lagunas?

¿Sus resultados científicos son suficientes para evaluar y medir todos los riesgos asociados a la ciencia forense?

Podríamos otorgar una respuesta ventajista común a todas las cuestiones planteadas: la ciencia forense es autosuficiente puesto que asume su rol edi- 
ficado en años de estudio, experiencias y resultados. Situémonos ahora en el lado opuesto, tratando la misma ciencia desde una visión problemática, en este caso, si hallamos respuestas, habremos ejercido un nuevo filtro sobre su autosuficiencia, es decir, generaremos lo que podríamos denominar una minimización de riesgo, ya citada en la introducción del presente trabajo, prestigiando su rigor, su carácter y su credibilidad y por qué no decirlo, prestigiando a los profesionales que se ocupan de ejercerla, la respuesta aplicada significará lógicamente la identificación de los indicios problemáticos, habremos ido directos al inicio de toda solución, el conocimiento del factor de riesgo, a partir de aquí, el procedimiento ha de culminar en la propuesta de acciones que posibilite la erradicación o minimización problemática, teniendo en cuenta como ya dijimos, que riesgos menos numerosos no significan obviamente que también sean menos graves, luego la relevancia dependerá del propio problema o riesgo emergente.

Sometamos este tipo de cuestiones a discusión mediante la exposición general de una idea, de un proyecto de trabajo basado en la planificación, ahondar primariamente sobre un camino innovador como es la producción de inteligencia al servicio de la ciencia forense, es decir, exponer un marco teórico bajo el prisma preventivo con el fin de anticipar el daño que produce el hecho problemático, o más bien, exponer una situación que puede derivar en el daño, redireccionar herramientas aplicadas en el análisis de inteligencia, que sean capaces de sistematizar la obtención de información del «caso problema» sometido a ensayo, su análisis y su difusión a quien haya de tomar las decisiones como respuesta al planteamiento problemático inicial.

\section{Inteligencia y Ciencia Forense: estrategia y prospectiva}

Es una realidad plausible y un hecho constatado que el estudio prospectivo o la planificación estratégica alimentada por información debidamente valorada, analizada, integrada e interpretada constituye un elemento esencial para minimizar la incertidumbre, al posibilitar la toma de decisiones importantes, necesarias y/o críticas. Estar informado bajo el principio de oportunidad (una información no oportuna es inservible) constituye en sí mismo un elemento de poder no desdeñable para cualquier Nación. No por ello, disminuyendo los escalones decisorios pierde su valor, la empresa, la universidad, la institución académica..., deben reforzar sus debilidades o potenciar su propia fortaleza ya existente, y no solamente ha de ir dirigida a aumentar la misma, sino a evitar su depreciación o la perdida de lo ya conseguido, estimando la línea más débil para evitar su fuga, y por consiguiente, eliminando la reducción del riesgo. Si fijamos la alerta más pronto que tarde, antes trabajaremos sobre la solución desde los dos frentes citados.

La estrategia ha de ir dirigida precisamente a la anticipación para minimizar consecuencias no deseadas y previsibles a medio o largo plazo con lo que ya conocemos en el corto plazo (presente). Si en el momento de realización de un trabajo de este tipo, hallamos posibles factores de riesgo, las medidas de acción propuestas serán más efectivas y acertadas, puesto que ya poseeremos los indicios problemáticos que nos hagan iniciar la necesidad de inteligencia para exponer dicha situación de riesgo y aplicar de este modo, las posibles vacunas preventivas, conformando un marco de actuación futuro de peso. Por lo tanto, la estrategia «anticipadora» ha de encaminarse al hallazgo de aquellos factores que puedan debilitar, en el caso que nos ocupa, la ciencia forense.

En definitiva, se trata de plantear el problema presente, o en caso de no existir, plantear problemas futuros con rango de hipótesis (estimativas, predictivas), a fin de anticipar soluciones estratégicas. No se trata evidentemente de inventar situaciones críticas, se trata de dirigir, obtener, elaborar y difundir he- 
chos que pueden revestir importancia si no somos capaces de planificar nuestra estrategia en tiempo y de forma oportuna. Introducimos otro campo muy asociado a la estrategia, la prospectiva y a su vez, asociadas ambas a la inteligencia.

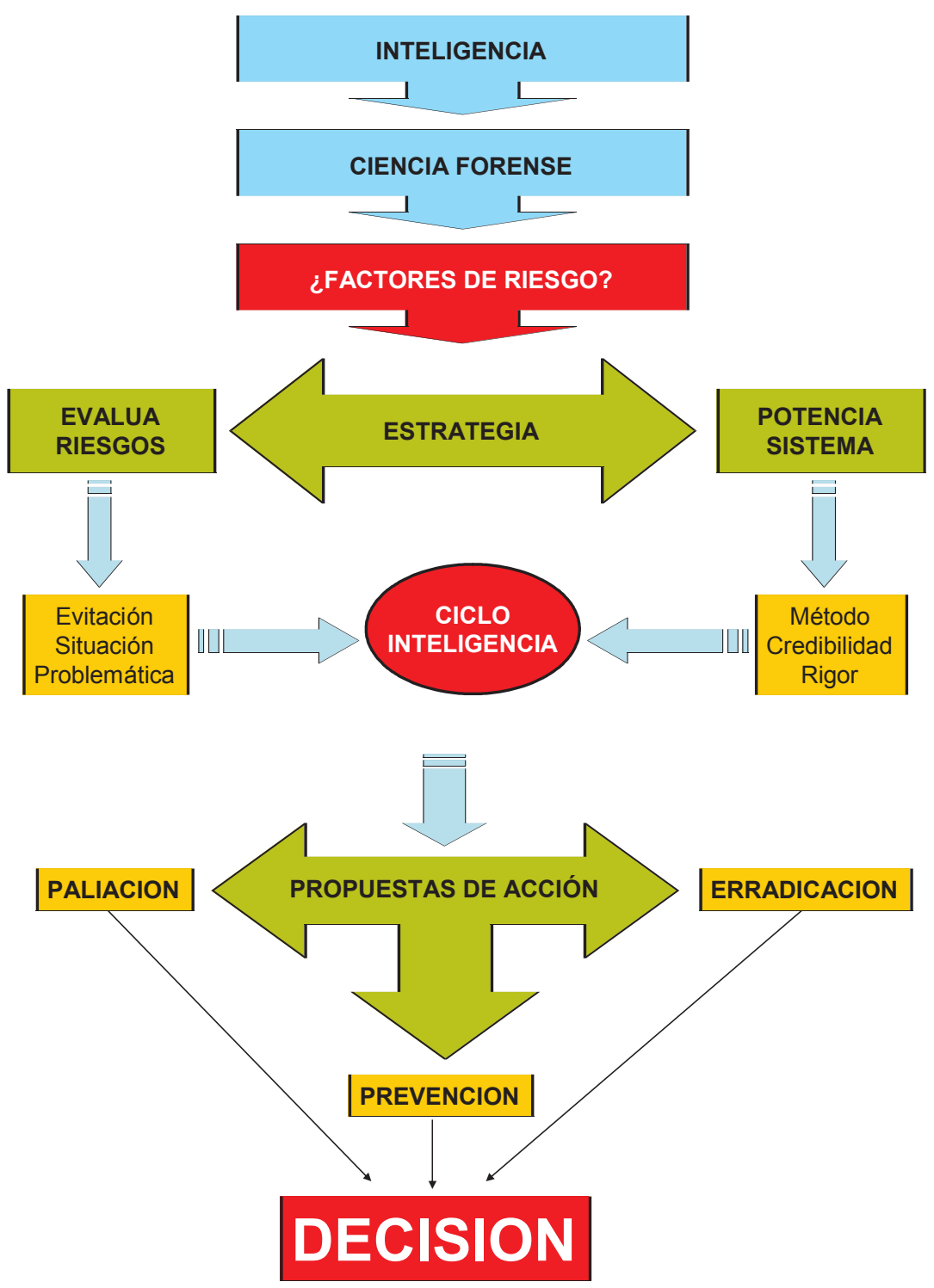

Conceptuemos la prospectiva y su relación con la estrategia, reflejando así mismo su valor en la inteligencia.

"La prospectiva es la disciplina que estudia el futuro para comprenderlo y poder influir en él» (7)

"I...de todo lo que podemos llegar a saber sobre el futuro lo menos interesante es el qué pasará, lo realmente relevante es el cómo y, sobre todo, el porqué. En efecto, la prospectiva busca entender cuales son los mecanismos causales y la secuencia de hechos que puede llevar a que sea uno, de entre todas las posibilidades, el futurible que acaba sucediendo» (8)

"...la función principal de la prospectiva es ayudarnos a decidir (en el presente) sobre situaciones respecto a las cuales hay una gran incertidumbre» (9)

«...la prospectiva...nos permitirá buscar la información disponible, trazar la 
conexión entre datos sueltos y analizar los posibles efectos de cada línea de acción» (10)

"El rol fundamental de la estrategia en un proceso de toma de decisiones es integrar la diagnosis; es decir, valorar la capacidad del sujeto para llevar a cabo un curso de acción o de encajar sus efectos» (11)

"Debemos incorporar factores cualitativos y la estrategia de los actores. Ya que los aspectos normativos pueden ser más determinantes que los estructurales a la hora de modelar el futuro; por lo tanto, ignorarlos es arriesgarse a fallar de la manera más estrepitosa» (12)

«...prospectiva e inteligencia tienen mucho en común...: ambas disciplinas tienen una función similar: intentar formular información o conocimiento para poder decidir con más fundamento» (13)

"...lo que no se puede es dejar que los acontecimientos nos superen e ir a remolque de ellos, adaptando las decisiones a las imposiciones del momento, sino que hay que adelantarse a los acontecimientos. Se vuelve a insistir, por su trascendencia, que ése es el verdadero valor de la inteligencia estratégica.

...y esta inteligencia estratégica debe ser capaz de ofrecer una conclusión prospectiva que valore entre lo posible y lo deseable» (14)

"...en ello se basa todo análisis estratégico, en adelantarse a los riesgos y amenazas presentes para evitar sus consecuencias futuras» (15)

\section{Ejemplos de inferencia Inteligencia - Ciencia Forense}

\section{Ejemplo 1 (Bolonia)}

Podemos considerar que uno de los pilares básicos del proceso científico es el rigor. Este se asienta entre otras circunstancias, en una formación específica que otorga credibilidad al método, al proceso, en definitiva, a la pericia forense. Se construye pues, mediante cimientos asentados en la esencia académica: la formación, la especialidad forense, el perfeccionamiento, la excelencia, la práctica y la exclusividad que otorga su pericia, de la cual será demandante en una gran mayoría de sus casos, la autoridad judicial. La idea general por lo tanto, será sistematizar el análisis de inteligencia válido para la paliación, minimización y solución de graves formas de delincuencia y extrapolarlo a la ciencia forense, con el fin de establecer seguridad sobre posibles fugas que a medio o largo plazo puedan resultar problemáticas, así como ahondar en la búsqueda de aquello que pueda debilitar la acción forense.

En el presente ejemplo, la estrategia general sería la de mantener y fomentar la educación y formación forense, validando con datos siempre objetivos la necesidad indispensable de contar con especialistas bien formados, provocando esa ética corporativa (organizacional) sobre la convicción común de llevar a cabo la estrategia pre-planificada en la parte afectada y con los datos que apoyen que el método académico actual puede ser beneficioso o perjudicial en función de su perspectiva comparada, no sólo académica, incluso poder someter a contradicción dicha comparación con el nivel de criminalidad de un país o región que será quien exponga la necesidad de la acción formativa que otorga un buen proyecto largamente alimentado por una Escuela de referencia y con una carga lectiva acorde a la exigencia de la propia ciencia forense.

Es decir, consideraremos como hipótesis viable que la reducción del número 
de horas en la formación forense de los alumnos, puede resultar un factor de riesgo para la propia ciencia forense y como consecuencia de éste, podría contribuir y/o constituirse en un factor de debilitamiento del sistema, asociando también su cualificación y su valor en la intervención de la ciencia forense en muchos de los casos que conforman la estadística delincuencial de un país o de una región, es decir, elevar su jerarquía interventora en función del nivel criminal, en relación delitos - población en riesgo o reduciendo su comparación al tipo de delito - intervención de la ciencia forense - población en riesgo.

Expuesta la idea, el siguiente paso, sería dar contestación a las numerosas interrogantes que surgen del objeto - problema, seguidamente quedaría elaborar en el marco de la inteligencia estratégica, el establecimiento de posibles soluciones de tipo paliativo o preventivo mediante el método de trabajo, el ciclo de inteligencia que posteriormente veremos.

¿Constituye el Espacio Europeo de Educación Superior (Plan Bolonia) un problema en el ámbito académico respecto a la formación en la ciencia forense?

¿Puede constituirse en factor de riesgo?

¿Es viable la hipótesis formulada?

¿Existe percepción académica sobre el problema?

¿Pueden verse afectadas otras instituciones relacionadas con la ciencia forense?

¿En el caso de considerarse como problemático el Plan o parte de él, cómo afronta la Escuela de Medicina Legal las posibles vías de solución?

¿Y la Universidad?

¿Existen soluciones paliativas que minimicen el daño o el riesgo?

¿Existen soluciones preventivas modificativas?

¿Son eficaces?

¿Existe perspectiva comparada con otras instituciones académicas españolas en el ámbito de la ciencia forense?

¿Existe perspectiva comparada con otras instituciones académicas europeas en el ámbito de la ciencia forense?

¿Existe perspectiva comparada con otras instituciones relacionadas con la criminalidad?

¿Podemos por tanto identificar elementos de riesgo?

¿Podemos cuantificar su incidencia?

¿Podemos cualificar la gravedad independientemente de su incidencia?

¿Cuál es el nivel real de la problemática supuesta? ¿Se conoce?

¿Es asumible su existencia?

¿Puede crear dudas razonables en la formación de expertos?

¿Puede restar credibilidad y rigor la menor cualificación de expertos?

¿La potenciación de un plan y unas circunstancias limita otras?

¿Una decisión presente (reducción formativa) puede ser contraproducente en decisiones futuras (convicción judicial)?

Estas y otras cuestiones son a las que habría de dar respuesta, organizar la planificación de la estrategia, diseñar la información que ha de recopilarse, obtener dicha información, evaluar la misma y convertirla en inteligencia, exponiendo las inferencias (hipótesis, conclusiones, predicciones y estimaciones) aplicables al caso, conformando finalmente concretas y explícitas propuestas de acción para el apoyo de la decisión en la erradicación de la amenaza o del riesgo.

\section{Ejemplo 2 (Ausencia de cuerpo de víctima en delitos graves)}

El artículo 138 del vigente Código Penal, recogido en el Libro II, Título I sobre el homicidio y sus formas establece que: 
«El que matare a otro será castigado, como reo de homicidio, con la pena de prisión de diez a quince años».

El artículo 139, en cambio, establece que:

"Será castigado con la pena de prisión de quince a veinticinco años, como reo de asesinato, el que matare a otro concurriendo alguna de las circunstancias siguientes:

1. Con alevosía.

2. Por precio, recompensa o promesa.

3. Con ensañamiento, aumentando deliberadamente e inhumanamente el dolor del ofendido.»

Estos son dos de los delitos más graves recogidos en nuestro actual Código Penal. La primera acción de la instrucción judicial y su investigación derivada, será recoger como establece la Ley de Enjuiciamiento Criminal en su capítulo II (cuerpo del delito), las "armas, instrumentos o efectos de cualquiera clase que puedan tener relación con el delito y se hallen en el lugar en que éste se cometió».

Dice también en su artículo 335 qué «siendo habida la persona o cosa objeto del delito, el Juez Instructor describirá detalladamente su estado y circunstancias y, especialmente, todas las que tuviesen relación con el hecho punible».

¿Y qué ocurriría si no aparece el cuerpo objeto del delito o cadáver de la víctima?

El artículo 343, lejos de aclarar la situación y despejar dudas, enturbia más si cabe el supuesto citado, puesto que constata que:

"En los sumarios a que se refiere el artículo 340 (muerte violenta o sospechosa de criminalidad), aun cuando por la inspección exterior pueda presumirse la causa de la muerte, se procederá a la autopsia del cadáver por los médicos forenses, o en su caso por los que el Juez designe, los cuales, después de describir exactamente dicha operación, informarán sobre el origen del fallecimiento y sus circunstancias».

El citado artículo 340 certifica la necesidad de la autopsia para conocer el origen del fallecimiento y sus circunstancias, es el propio legislador quien aboga por una segunda prueba específica, admite inicialmente la generalidad (aún cuando por la inspección exterior...) para plantear la particularidad de la prueba certera, el estudio del cadáver, intenta evitar la duda contrastando la primera impresión del hecho - consecuencia y de la causa - efecto producida. Si del examen inicial de la sospecha de muerte, poseyendo el cuerpo objeto del delito, asume como protocolo necesario la realización de autopsia, ¿qué ocurriría entonces si no hallamos el cuerpo de la víctima? Inicialmente parece contradictorio y aún más dubitado.

Como ya he reflejado, el tipo penal de asesinato que podemos caracterizarlo como el agravante del homicidio se halla sometido a la interpretación y consideración de tres parámetros muy claros; la alevosía, el ensañamiento y el precio, recompensa o promesa, circunstancias estas diferenciadoras del homicidio. Conocer esa agravación de la acción criminal hace necesario el estudio forense del cuerpo. Sin víctima se facilitará la acción de la defensa, salvo que la investigación criminal (policial) clarifique explícitamente con suficiente carga probatoria y/o indiciaria externa dicha diferenciación, esencial en este tipo de casos. La autoinculpación del supuesto autor no es suficien- 
te si no va acompañada de esas pruebas o indicios externos citados y siempre con la debida cautela que impone la no existencia de cuerpo.

Es decir, la cuestión diferencial entre ambos delitos radica en la forma y modo de matar. Sin el cadáver, determinar o inclinarse por uno u otro tipo penal parece bastante difícil e incluso atrevido. Por lo tanto hemos de cuestionar nuevamente ¿sin cadáver hay delito?

"La condena no está excluida de que aparezca el cadáver, sino de que se comprueben los hechos».

Sin embargo, no es habitual que los tribunales condenen a alguien por asesinato sin el cuerpo. Los tribunales son bastantes cautelosos. Tienen que tener la convicción plena de que está muerta, porque no se quieren arriesgar a condenar a alguien por el asesinato de una persona que puede aparecer viva años después». (16)

Por otro lado, la Ley de Enjuiciamiento Criminal tampoco es determinante al respecto, no exige cadáver sino pruebas razonables de que el hecho se ha producido y pruebas que incriminen al autor o autores. ¿Podemos por tanto considerar el cadáver un elemento de prueba más? Y en el caso de no ser hallado, ¿el resto de pruebas implicatorias podría encausar al sospechoso?.

Objetivamente, sin cuerpo y sin autopsia quizás no haya homicidio, quizás no haya asesinato, quizás no haya otros delitos asociados (agresión sexual) y quizás sí haya detención ilegal y quizás sí haya encubrimiento siempre y cuando tengamos sospechoso y esas sospechas demuestren al menos, que sí colaboró en la retención de la víctima contra su voluntad antes de la desaparición del cuerpo o que sí participó en otras acciones (traslado, ocultación del cuerpo).

Evidentemente, la libre apreciación de la prueba depende del Tribunal. Ahora bien, condenar por homicidio o asesinato sin el cuerpo, sin el examen forense del mismo, «prueba evidente de su diferenciación parece complicado». La trascendencia social de un caso puede inclinar la balanza, pero quizás estemos ante un nuevo riesgo o amenaza para la ciencia forense como órgano consultivo, como órgano pericial, como disciplina de insustituible, imprescindible e indispensable valor en el análisis forense.

El presente caso solo es un ejemplo real y de enorme actualidad en estos momentos, es por ello que:

¿Casos como éste puede debilitar la esencia forense?

¿Disminuye su valor?

¿Disminuye su potenciación pericial?

¿Se deprecia su aportación?

¿Perjudica su rigor?

¿Enturbia su credibilidad?

¿Se resta importancia a su labor?

¿La respuesta judicial puede minimizar la necesidad forense en casos de este tipo?

¿La trascendencia social de un caso puede influir negativamente a la hora de determinar la necesidad forense?

¿La ciencia forense (Institutos Anatómicos, Escuelas de Medicina Legal,

Profesionales, Colegios Oficiales) pueden o deben posicionarse como

órganos consultivos en casos como este?

¿Cómo valora o interpreta la ciencia forense la Ley de Enjuiciamiento

Criminal en casos de este tipo?

¿Puede determinarse sin existencia de cuerpo de víctima la calificación del 
tipo penal?

¿Es acertado desde la visión forense juzgar un caso sin la existencia del cuerpo objeto del delito?

¿Juzgar sin cuerpo puede crear la duda razonable del Tribunal?

¿Qué pruebas externas pueden validar una condena positiva sin la existencia del Cuerpo?

¿La presencia del sospechoso en el escenario criminal y su impronta biológica puede sustituir la necesidad forense del análisis del cuerpo? ¿Dichas pruebas serían suficientes para catalogar un delito como homicidio o asesinato?

Estas y otras cuestiones pueden marcar una situación de riesgo en la ciencia forense, y como hemos visto en los dos ejemplos citados, con distinto perfil pero con un marcado carácter estratégico, el primero más predictivo, el segundo más conclusivo, ponen de manifiesto que la situación problemática puede ser más global y de más amplio espectro, con afectación de mayores actores implicados, cuya influencia indirecta puede interferir directamente en la ciencia forense.

\section{Método de trabajo: ciclo de inteligencia}

Tradicionalmente, desde hace más de tres mil años, la información de carácter secreto ha sustentado el conocimiento como necesidad ultradefensiva de la amenaza. No hay mayor poder que dirigir una estrategia per-se que paralice o minimice el riesgo conocido antes de su producción. Con el paso del tiempo, el volumen de información que acumulaban los servicios secretos de los estados hacía necesario someter la misma a un proceso que puliera lo realmente válido y descifrara aquello que parecía inviable, había que ordenar lo desordenado, debía nacer un método capaz de procesar ese desorden, dotar a ese método de carácter científico, y otorgar con rigor un resultado "decisorio», pues el fin era y es precisamente poner en conocimiento la existencia de la amenaza primero y en segundo lugar, dirimir la influencia en la decisión que haya de tomarse y por quien haya de tomarla. Rigor asentado en quién y como lo hace.

Ese proceso, ese método de trabajo, fue denominado "ciclo de inteligencia», un ciclo formado por cuatro fases y subfases que posteriormente veremos.

\section{Según Michael Warner (2006) (17) «para llegar a una teoría lo primero que} se necesita es una definición».

Efectivamente, antes de adentrarse mínimamente en la explicación de un método de trabajo, debemos conceptuar previamente una serie de parámetros para comprender la teoría que llevará a la práctica, en el caso que nos ocupa, creo necesario apuntar una serie de definiciones previas de carácter más general, antes de profundizar en la disquisición de cada una de las fases y subfases que componen el ciclo de inteligencia en nuestro país. Deseo marcar este matiz dado que en EE.UU. por ejemplo, tanto la nomenclatura de sus fases como su subdivisión es diferente, no obstante el producto final será el mismo, la información convertida en inteligencia que es lo verdaderamente importante.

Previamente, deseo apuntar que las dos grandes divisiones de la inteligencia actual son la inteligencia estratégica y la inteligencia táctica, asociadas también al tipo de elaboración ( $3^{a}$ fase del Ciclo). Ambas se diferencian o se caracterizan por su inmediatez: la primera actúa a medio o largo plazo y la segunda a corto plazo. Tanto una como otra pueden alimentarse de forma continua, por lo tanto siempre estarán sometidas a contraste, evolución, ampliación, modificación o reducción. La segunda, la inteligencia táctica se 
hallará sujeta y limitada a la operatividad de la explotación, luego los campos de aplicación son distintos.

Por ejemplo, la ciencia forense como campo de aplicación estratégico podría colaborar en la creación de un diagnóstico de riesgo sobre los factores positivos que influyen en la captación y reclutamiento de futuros terroristas con el fin de proponer acciones de paliación o solución. Aquí podría tener cabida aquellos estudios relacionados con el comportamiento y la conducta individual y como éstos pueden influir en el comportamiento o conducta grupal (sociología de la violencia).

Tácticamente es evidente que la ciencia forense ejerce y colabora habitualmente en la investigación y resolución de muertes sospechosas de criminalidad o caracterizando organizaciones criminales o perfiles de autoría criminal, aportando su análisis y conocimiento a la investigación criminal en curso.

Vista la puntualización anterior, vayamos pues directamente a citar textualmente aquellos conceptos generales, no por ello menos importantes:

Noticia (18): «Materia prima o elemento mínimo de la que se alimenta la información global de un determinado asunto».

Información (19): «Conjunto de noticias que pueden estar o no relacionadas entre sí. Tiende a aplicarse a noticias interrelacionadas, es decir, que se refieren a un mismo tema».

Información-bis (20): "Conjunto de datos sin procesar para su conversión en conocimiento. Es también la acción y el efecto de dar noticia de algo. En consecuencia, para un servicio de inteligencia es información todo dato, noticia o documento no evaluado y analizado que tiene un interés potencial como materia prima para la producción de inteligencia».

Fuentes de información (21): "Conjunto de instrumentos que se utilizan para satisfacer las necesidades y demandas de información de un usuario. De las fuentes de información emanan los datos que sirven de origen y base para la elaboración de conocimiento».

Inteligencia (22): «Producto que resulta de la evaluación, la integración, el análisis y la interpretación de la información reunida por un servicio de inteligencia».

Inteligencia actual (23): "Tipo de inteligencia cuyo fin es satisfacer los requerimientos de inteligencia puntuales y concretos de una organización. También detecta y advierte sobre indicios de riesgos».

Necesidad de inteligencia (24): «Materia general o específica sobre la que se requiere inteligencia. Reconocimiento por un usuario de que algún aspecto de su estado de conocimiento de una realidad concreta es incompleto porque le falta información. Por tanto, por medio de una adecuada reflexión y análisis, se puede determinar qué información se debe adquirir y procesar para resolver un problema o tomar una decisión.

Pero no toda necesidad de inteligencia da lugar a una pregunta concreta al servicio de inteligencia. La conversión de una necesidad de inteligencia en una demanda efectiva depende de tres factores: el propio reconocimiento por el usuario de su necesidad, la importancia y el valor que el usuario otorgue a la satisfacción de esa necesidad y la percepción por el usuario de la probabilidad de que el sistema de información responda a su pregunta». 
Riesgo (25): «La conversión de una amenaza en un daño posible y próximo debido a la evolución de una situación. El riesgo es propio de situaciones caracterizadas por la incertidumbre. Los servicios de inteligencia se ocupan de la detección y el análisis de la evolución de las variables que permiten que un daño o un fracaso potenciales se puedan convertir en un daño o fracaso reales. Cuando las variables no toman un único valor sino varios, pero la probabilidad de ocurrencia de esos valores es exactamente conocida, nos encontramos en un contexto de riesgo».

\section{Ciclo de Inteligencia}

«Se entiende por Ciclo de Inteligencia la secuencia mediante la cual se obtiene información, se transforma en inteligencia y se pone a disposición de los usuarios. El Ciclo de Inteligencia consta de cuatro fases: dirección, obtención, elaboración y difusión» (26).

Podemos decir que es un proceso cronológico mediante el cual, la información global o específica previamente definida por la propia necesidad de inteligencia, finaliza con un producto o resultado del que se deduce algo: conocimiento útil para el apoyo de la decisión, que puede servir exclusivamente al órgano que la ha planificado o elevarla a órganos superiores que la hayan demandado.

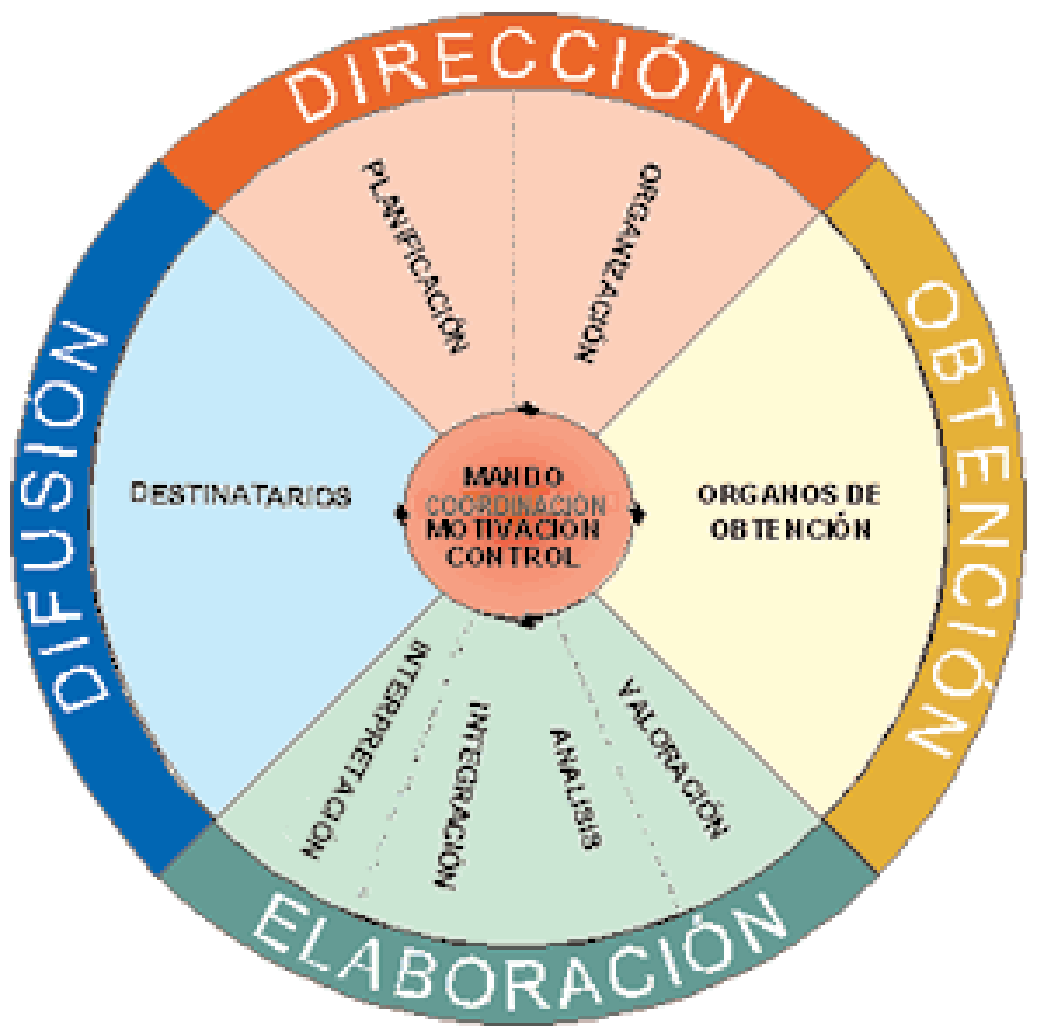

Primera Fase: Dirección. «Durante la fase de dirección se determinan las necesidades de inteligencia, se prepara un plan para su obtención, ser organizan los medios y se efectúa el mando, coordinación y control de todos ellos.

En esta fase cobran especial relevancia las denominadas funciones directivas, que son las siguientes: planificación, organización, motivación, mando, coordinación y control, manteniéndose las cuatro últimas durante el desarrollo de todo el ciclo». (27)

Segunda Fase: Obtención. «En esta fase se realiza la explotación de las 
fuentes de información por los órganos de obtención y la entrega de esta información al correspondiente equipo de elaboración para la producción de inteligencia». (28)

Tercera Fase: Elaboración. «Es la fase del Ciclo de Inteligencia en la que se produce la transformación de la información en inteligencia al someterla a un proceso apropiado, mediante la valoración de la pertinencia, oportunidad, fiabilidad y exactitud de las noticias e informaciones recibidas sobre cada una de las actividades seguidas, el análisis de las mismas, la integración con la inteligencia disponible y la interpretación del conjunto. Esta fase se divide en cuatro subfases: valoración, análisis, integración e interpretación».

- Valoración: primer filtro, mide la información y la distribuye.

- Análisis: extrae y agrupa datos.

- Integración: relaciona los datos extraídos con la inteligencia disponible sobre el mismo asunto. Se confecciona un análisis global.

-Interpretación: se formulan hipótesis, conclusiones, predicciones y estimaciones sobre el conjunto de la información integrada. El resultado producido ya es inteligencia.

Por otro lado, es necesario apuntar que «no se puede establecer un proceso de elaboración estricto que reuna las condiciones de universalidad que sería deseable, ya que el proceso será mas o menos complejo según las características de la información y del nivel del Servicio u Órgano de inteligencia». (30)

Cuarta Fase: Difusión. «Es la fase en la que se efectúa la distribución segura y oportuna de la inteligencia en la forma adecuada y por los medios apropiados a aquellos que la necesitan. La difusión es la fase final del Ciclo de Inteligencia». (31)

\section{Creación de un modelo en la Ciencia Forense}

\section{Dirección}

1. Planificación de la necesidad de inteligencia:

- Qué información necesitamos obtener

- Donde la podemos obtener

- Cómo podemos obtenerla

1.1. Debilitamiento del sistema: conocer sus causas, reducir sus consecuencias.

1.2. Potenciación: aumentar credibilidad y rigor con el fin de contrarestar su debilitamiento. Posicionamiento como órgano consultivo.

2. Organización para llevar a cabo la obtención de información objeto de la necesidad planificada:

\subsection{Materialización:}

- Medios humanos y materiales

- Doctrina para la obtención

- Seguridad en la obtención 
Recopilar información en relación con la necesidad de inteligencia. Utilización de fuentes de obtención.

1. Hallar posibles factores de riesgo indicativos de debilitamiento.

2. Buscar datos cuantitativos que caractericen el factor o factores de riesgo que puedan conllevar a una situación problemática.

3. Buscar datos cualitativos que refuercen la gravedad del riesgo para evidenciar la necesidad en la búsqueda de soluciones.

4. Hallar actores implicados: directos e indirectos.

5. Exponer datos propios y someterlos a contradicción con aquellos datos que alertan del riesgo o amenaza.

6. Obtener datos del mismo tipo en otras instituciones nacionales para someterlos a comparación.

7. Obtener datos del mismo tipo en otras instituciones europeas para someterlos a comparación.

8. Reforzar el propio sistema dotándolo de una cualificación especial e importante, determinando su fiabilidad y rigor:

- Capacitación forense (credibilidad de la institución como órgano especializado en el ámbito local, nacional o internacional), credibilidad de su laboratorio, credibilidad de sus profesionales, experiencia consultiva como órgano especializado...).

- Marco legislativo y normativo que evidencie la necesidad de su actuación forense ante los órganos judiciales.

- La importancia per-se de la ciencia forense en la determinación de sus conclusiones en aquellos casos que revisten una gravedad especial (asesinato, homicidio, agresión sexual...).

- La importancia de la ciencia forense en la demostración de la prueba material como apoyo a la investigación criminal (policial y judicial) de aquella delincuencia de carácter más grave (terrorismo y crimen organizado).

- Cooperación recíproca de la inteligencia y la ciencia forense ante graves casos de delincuencia antes aludidos (ej: de carácter estratégico: determinación de perfiles criminales de riesgo para anestesiar la ideología radical - captación de terroristas - apología-efecto propagandista de captación...etc. ó de carácter táctico: búsqueda de nuevos indicios o corroboración de los ya supuestos, recopilados en la fase de explotación). - Asociar su importancia a la estadística de delitos de carácter grave que se producen en su ámbito de actuación (ej: muertes violentas de carácter local) - Extrapolar su importancia a la estadística de delitos de carácter grave que se producen fuera de su ámbito de actuación (ej: muertes violentas de carácter nacional).

- Número de dictámenes forenses realizados.

- Número de procedimientos judiciales en los que haya intervenido como órgano consultivo.

- Número de profesionales formados en su Escuela de Medicina Legal. - Importancia de sus resultados en las resoluciones procesales en las que intervienen (inculpación o absolución).

- Importancia de sus resultados en la determinación de aspectos jurisprudenciales de notable relevancia (sentencias cuyo valor forense en la determinación de la prueba halla sido parte importante de la resolución o fallo).

- Otras.

Elaboración

1. Valorar toda la información recolectada y desechar aquella que sea inservible.

2. Analizar, extraer y agrupar datos de interés. 
3. Integrar la información procesada y analizada con el conjunto ya disponible sobre el objeto o ciclo investigado.

4. Interpretar mediante el establecimiento de relaciones: conclusiones, hipótesis, estimaciones y predicciones.

Determinar todo lo anterior en concretas propuestas de acción de tipo reactivo, paliativo o preventivo que faciliten y ayuden a la decisión.

\section{Difusión}

Pueden darse varias opciones, dependiendo del destino final de la inteligencia producida.

1. En primer lugar, siempre a quién haya planificado la necesidad de inteligencia (Dirección), que determinará:

- Finalización del proceso (consumo propio de la inteligencia obtenida).

- Distribución a los escalones inferiores dentro del propio órgano de inteligencia.

- Continuación del proceso (difusión externa).

2. En segundo lugar, a quién haya demandado la necesidad de inteligencia, que puede ser coincidente con la Dirección (planificación y organización) y por lo tanto se trataría de una difusión compartida con el órgano superior demandante, o bien, trasladar la inteligencia obtenida a otro órgano pertinente, distinto al del ciclo trabajado (cooperación/coordinación).

\section{Conclusiones}

«...la temática criminal abordada desde su investigación, ha de provocar la reflexión más allá de su cometido eminentemente reactivo y paliativo, la conciencia preventiva, se antoja necesaria y vital en un mundo cada vez más complejo, sobreabundante y repleto de continuas amenazas en constante expansión y evolución». (32)

Es esa conciencia preventiva la que enmarca el presente trabajo, Inteligencia y Ciencia Forense pueden beneficiarse de un intercambio de planteamientos y experiencias mutuas, en definitiva, doctrina común al servicio de un bien común.

Volviendo al inicio del presente trabajo, daremos ahora respuesta a las primeras interrogantes planteadas:

¿Podemos predecir un perfil de riesgo en la ciencia forense?

- La ciencia forense como otras disciplinas no está exenta de riesgos.

- El perfil de riesgo dependerá de la situación de incertidumbre.

¿Podemos estimar sus factores problemáticos?

- Teniendo en cuenta la evolución de "variables sospechosas", podemos estimar un daño posible y previsible.

¿Podemos evitar consecuencias no deseadas?

- Si identificamos los factores de riesgo potenciales en tiempo oportuno y prevemos líneas de acción futura, ante la posibilidad de su desarrollo problemático, podremos evitar consecuencias negativas derivadas.

- La prevención por encima de la reacción. 
¿Podemos identificar sus actores implicados?

- Todo factor de riesgo como aquellos que pueden incidir en la ciencia forense, requiere de actores necesarios, en ese contexto de riesgo ambos irán relacionados, si identificamos unos, identificaremos los otros.

¿Podemos exponer una idea de trabajo para la evaluación de riesgos en la ciencia forense?

- El presente trabajo es un ejemplo de ello, la minimización de riesgo requiere previamente de su evaluación con el fin de evitar el debilitamiento del sistema o potenciar el mismo, otorgando un valor asegurado mediante la credibilidad comprobada y el rigor exclusivo.

¿Es posible extrapolar metodología de inteligencia para su uso en la ciencia forense?

- La presente monografía es una prueba de ello, no sólo es válido para la ciencia forense, cualquier organización que se halle expuesta a una situación de incertidumbre y tenga necesidad de conocer, podrá poner en práctica la metodología de inteligencia para decidir con mayor objetividad.

¿Y la ciencia forense, puede intervenir y coadyuvar a su vez con la inteligencia?

- Evidentemente, la psicología criminal, la psiquiatría forense, la criminología o la sociología pueden aportar conocimiento respecto a una amenaza o riesgo en expansión y evolución.

En definitiva, como bien dice el profesor Jordi Serra, en concordancia también con el planteamiento y desarrollo general que he deseado otorgar al presente trabajo,

«...si se prevé algo negativo, hay que trabajar para evitarlo; sí se vislumbra algo positivo hay que hacer lo posible para asegurarlo». (33)

\section{Bibliografía}

1. Jesús I. FernÁndez SÁnChez, 2009. Investigación Criminal. Editorial Bosch.

2. Gustavo Díaz Matey, 2008. Revista de Análisis y Prospectiva, $n^{0} 4$ - 2008.

3. Diego Navarro Bonilla, 2009.

4. Fernando Velasco, 2008. El País 11/11/2008

5. LuÍS dE LA CORTE IBÁÑEZ, 2008. El Imparcial.

6. I Congreso Nacional sobre Inteligencia, 2008.

7. Gastón Berger. Centro de Estudios Prospectivos. Francia.

8. JoRdi SeRRA del PINO, 2008. La prospectiva y la investigación del futuro.

9. JoRd SeRRA del PINO, 2008. La prospectiva y la investigación del futuro.

10. JoRdi SeRRA del PINO, 2008. La prospectiva y la investigación del futuro.

11. JoRd SeRRA del PINO, 2008. La prospectiva y la investigación del futuro. 
12. JoRd SeRRA del PINO, 2008. La prospectiva y la investigación del futuro.

13. JoRd SERRA del PINO, 2008. La prospectiva y la investigación del futuro.

14. Pedro Baños Bajo, 2008. Nuevos retos para la inteligencia estratégica en las operaciones complejas.

15. Jesús I. FeRnÁNDEZ SÁnCheZ, 2009. Investigación Criminal. Editorial Bosch.

16. Gonzalo Arroyo, 2009. (Magistrado de la Audiencia Provincial de Gerona).

17. Toward a theory of intelligence workshop report.

18. Jesús I. Fernández SÁnCHez, 2010.

19. Jesús I. Fernández SÁnchez, 2010.

20. Glosario de Inteligencia, 2007. Ministerio de Defensa.

21. Glosario de Inteligencia, 2007. Ministerio de Defensa.

22. Glosario de Inteligencia, 2007. Ministerio de Defensa.

23. Glosario de Inteligencia, 2007. Ministerio de Defensa.

24. Glosario de Inteligencia, 2007. Ministerio de Defensa.

25. Glosario de Inteligencia, 2007. Ministerio de Defensa.

26. Centro Nacional de Inteligencia (www.CNI.es), 2011.

27. Centro Nacional de Inteligencia (www.CNI.es), 2011.

28. Centro Nacional de Inteligencia (www.CNI.es), 2011.

29. Centro Nacional de Inteligencia (www.CNI.es), 2011.

30. Luís Peláez PIÑEIRo, 2008.

31. Centro Nacional de Inteligencia (www.CNI.es), 2011.

32. Jesús I. FERNÁNDEZ SÁNCHEZ, 2009. Inteligencia Criminal. Inteligencia y Seguridad: revista de análisis y prospectiva.

33. Jordi Serra del Pino, 2008. La prospectiva y la investigación del futuro.

Correspondencia Jesús fernández Sánchez c/ Alcalde Jose María Durán y

Pelayo, 27 Portal 2 - 3ㅁ

28914 Leganés (Madrid)

jifernandezsanchez@guardiacivil.es 